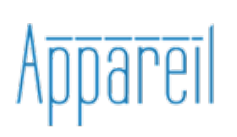

Appareil

$14 \mid 2014$

Esthétiques latino-américaines : penser à rebours

\title{
Action / Participation
}

L'art brésilien comme esthétique sociale

Stéphane Huchet

\section{(2) OpenEdition}

Journals

Édition électronique

URL : http://journals.openedition.org/appareil/2109

DOI : 10.4000/appareil.2109

ISSN : 2101-0714

Éditeur

MSH Paris Nord

Référence électronique

Stéphane Huchet, «Action / Participation », Appareil [En ligne], 14 | 2014, mis en ligne le 10 décembre 2014, consulté le 30 juillet 2020. URL : http://journals.openedition.org/appareil/2109 ; DOI : https:// doi.org/10.4000/appareil.2109

Ce document a été généré automatiquement le 30 juillet 2020

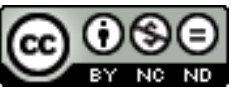

Appareil est mis à disposition selon les termes de la Licence Creative Commons Attribution - Pas d'Utilisation Commerciale - Pas de Modification 4.0 International. 


\title{
Action / Participation
}

\author{
L'art brésilien comme esthétique sociale
}

\author{
Stéphane Huchet
}

\section{NOTE DE L'ÉDITEUR}

Les textes portugais cités ont été traduits en français par Stéphane Huchet.

1 Au Brésil, l'art, considéré par certains « activistes » comme une action avec des règles de «participation » assez solidement établies, sollicite un public avec lequel il cherche à partager la responsabilité de reconfigurer momentanément un certain être-ensemble, une certaine expérience du commun. Ces notions connaissent une grande vogue actuellement et il est intéressant de constater que bien des manifestations artistiques les ont pré-figurées, notamment dans le Brésil des années 1960.

Dans le séminaire de Jean-Louis Déotte à la Maison des Sciences de l'Homme Paris Nord, voici quelques années, nous avons présenté un ensemble d'images de l'art brésilien des années 1960 aux années 2000. Notre choix s'est naturellement porté sur les dispositifs plasticiens qu'un nombre important d'artistes propose au public depuis quelques décennies. Tous ont pour finalité une "participation» du public. La "participation » est ainsi devenue une sorte de "genre", n'en déplaise à ceux qui considèrent les catégories artistiques anciennes comme actuellement obsolètes et illégitimes. Les noms : Lygia Clark et Hélio Oiticica pour les années 1960 ; Ernesto Neto et Ricardo Basbaum pour les années 2000, avec leurs installations qui invitent le public à s'asseoir, se coucher, s'étendre sur des supports souples, etc. Il est important de dire que les premiers artistes qui ont procédé à ce mixte d'art et d'action ont pensé dès l'origine leurs structures plasticiennes comme des supports de sensibilisation à une dimension nouvelle de l'art. Dans le mouvement «concrétiste» des années 1950 ou dans le mouvement "néoconcrétiste" des années 1960, il s'est toujours agi, par-delà les différences, de propositions plastiques à vocation structurante: l'information et la sensation construisent une expérience que son support bidimensionnel ou tridimensionnel ne change pas fondamentalement. Les concepts en vigueur chez les 
artistes brésiliens de ces années sont des concepts à la fois sémiotiques et phénoménologiques pensés pour l'édification d'une sensibilité commune singularisée. Rappelons leur perspective.

3 Historiquement, le concrétisme de São Paulo a une conception purement visuelle de la forme et prend rapidement une orientation rationaliste à travers le choix d'un modèle esthétique pensé comme scientifique. Selon le critique Ferreira Gullar, le concrétisme pictural n'a pas su aborder la question de la perception dans toute sa complexité et dans toutes ses extensions. Le néoconcrétisme, centré sur Rio de Janeiro, représente une sortie hors des ces limites. Il se veut, dit Gullar, une « symbologie tacite du corps $»^{1}$. La Première Exposition néoconcrète, en 1959, met en évidence la supériorité de la question des formes significatives par rapport aux jeux optiques. Le néoconcrétisme est double : il rompt avec la pragmatique sémiotique du concrétisme, mais il reconduit en même temps ce que les critiques d'art nomment « le projet constructif de l'art brésilien » à des fondements que le concrétisme aurait détournés de ses implications symboliques. Si les deux mouvances artistiques en question sont bien des manifestations du « projet constructif de l'art brésilien", le premier a échoué sur les grèves froides de l'illustration tandis que le second réalise un retour sur ses fondements structurants et une "recherche intuitive d'un nouveau langage symbolique $»^{2}$. En tant que "symbolique tacite du corps ", le néoconcrétisme cherche à déplacer le champ d'action vers la recherche d'effets esthétiques beaucoup plus complexes. Gullar maintient ses analyses à l'intérieur de catégories en usage à l'époque, mais il propose surtout une interprétation des travaux néoconcrets de la fin des années 1950 en termes de déplacements stratégiques par rapport au noyau dur concrétiste et de retour aux véritables fondements constructifs, pour mieux tracer un futur. Des catégories auxquelles l'histoire de l'art de cette époque nous a habitués, où l'on retrouve un ensemble constitué de psychologie de la perception, de phénoménologie et de formalisme, servent alors à situer les différences: Gullar croit ainsi nécessaire de rappeler comment la représentation classique gère son rapport à l'espace, afin de mieux la différencier de la projection moderne de la peinture vers l'espace tridimensionnel. Il repère clairement cette tension vers une tridimensionnalité externe dès les Relevos Bilaterais (Reliefs Bilatéraux) de Oiticica, autour de 1960, conçus comme "luminosité de la couleur $»^{3}$, présentant une «conception architectonique $»^{4}$ de la couleur, tandis que les Bichos de Lygia Clark, structures en perpétuelle "métamorphose ${ }^{5}$, opèrent un passage de l'image, du monde de l'image - dans son sens encore relativement traditionnel d'image bidimensionnelle prise dans les coordonnées conventionnelles du tableau - au " mirage $»^{6}$, une sorte de zone formelle dynamique et en état de modification constante selon la manipulation gestuelle à laquelle elle est soumise.

4 Un autre critique, Ronaldo Brito, écrivant une quinzaine d'années plus tard, complète l'analyse. À un concrétisme qui cherche à "transformer l'environnement social contemporain ${ }^{7}$, à agir sur le plan de l'information de masse à travers des processus sémiotiques, il oppose une pratique expérimentale autonome, la "marginalité » néoconcrétiste, qu'il considère même "apolitique " car elle ne se place ni dans le processus de consolidation sémiotique de la société de production industrielle, comme chez les concrétistes, ni dans la lutte politique affichée, comme ce fut le cas des Centres populaires de Culture du début des années 1960, ni, enfin, dans la qualification sensible du matériau industriel chez les sculpteurs Amilcar de Castro et Franz Weissmann, qui constituent selon lui l'aile la plus traditionnelle et conservatrice du néoconcrétisme. 
Pour Brito, le néoconcrétisme travaillait sans la pression du marché de l'art, sans visée commerciale, sans nécessité de reproduire des canons artistiques vendables, comme un espace de proposition d'idées, de formes et de processus inédits. Contre le pouvoir de l'appareil marchant, il pariait sur le pouvoir d'un appareil plus imperceptible, mais cristallisé circonstanciellement en des énoncés devant beaucoup à un mythe de l'art et en la foi en sa capacité médiatrice pour reconfigurer l'expérience. Au scientisme de l'art caractéristique du concrétisme des années 1950, à son « aspiration typiquement constructive à planifier l'environnement social selon les moules d'une rationalité modernisatrice $»^{8}$, à son idée d'art comme médium au service d'un plus ample réseau d'information, à sa conception de l'art concevant les images et les productions visuelles comme réponse artistique à une tendance «culturelle » et " économique » dominante, à sa conception de l'art comme esthétique collective de l'époque des mass media, Brito oppose dialectiquement le retour néoconcret à un humanisme "ontologique " qui se serait donné pour mission de porter plus loin et à de plus riches conséquences certaines découvertes concrétistes, ce qui signifie notamment la nécessité de penser l'expérience de l'espace en termes phénoménologiques. En tant que "symbologie tacite du corps", comme disait Gullar, le néoconcrétisme est dramatique et ontologique. Femme et " homme de culture ${ }^{9}$ autant, sinon plus, que "professionnels» de l'art, Clark et Oiticica auraient contribué à ce que cette avant-garde désinhibée redonnât un souffle aux « résidus idéalistes concernant le statut de l'art $»^{10}$. En vérité, Brito reproche déjà cela aux concrétistes : leur croyance en la possibilité d'une répercussion réelle de leurs manipulations sémiotiques sur la société, afin d'en soutenir les tendances progressistes, lui semble également reposer sur une croyance non questionnée en un idéal de l'art. Les visées du néoconconcrétisme sont moins utilitaires et surtout non intégrées à une idéologie techniciste, mais la foi en la force médiatrice et incarnante d'une autre loi, sauvage, celle de l'art sensoriel, le situent aux confins du même idéal. La symbolique néoconcrète $\mathrm{du}$ corps est mise au service d'une proposition plus expérimentale, sensible et participative qui concerne «l'ensemble de la relation de l'homme avec son environnement » car elle est une " activité culturelle globalisante ${ }^{11}$.

C'est la raison pour laquelle le critique Mário Pedrosa affirme dès 1966 la nécessité d'adopter de nouveaux critères de jugement face à un art «moderne »-c'est-à-dire "discipliné » par des «valeurs proprement plastiques »- qui se transforme en un art "post-moderne» dans lequel "les valeurs proprement plastiques tendent à être absorbées dans la plasticité des structures perceptives et situationnelles $»^{12}$. C'est aussi cette même année qu'Hélio Oiticica analyse dans le catalogue de l'exposition Proposta 66 , à São Paulo, le sens d'une « avant-garde » brésilienne, d'une " Nouvelle Objectivité » pensée en termes "d'ordres environnementaux », de nouveaux "objets » impliquant " toute l'échelle sensorielle $»^{13}$. Cette Nouvelle Objectivité est la première manifestation discursive d'un art de la participation à de «nouveaux objets perceptifs $»^{14}$ - et l'on voit que cette veine phénoménologique d'un art des sens, esthésiologique, est l'expression d'un désir irrépressible de l'époque - mais elle est aussi la manifestation de nouveaux objets "propositionnels d'où rien n'est exclu »" ${ }^{15}$ écrit Oiticica. Affirmant en 1968 la prépondérance très récente donnée aux valeurs de signifiance plutôt qu'aux valeurs expressives, Pedrosa y voit la marque d'une rupture avec un modernisme interprété par lui en termes d'œuvre "unique et privilégiée de l'artiste et du sujet $"^{16}$. Pedrosa nous offre ici un écho micro-logique et non développé de la conception greenberguienne de l'art moderne. Pedrosa écrivait en effet qu'«un langage extrêmement épuré s'était formé durant le siècle pour définir, isoler, exalter les 
suprêmes valeurs plastiques, expressives, esthétiques en chaque œuvre ${{ }^{17}}^{17}$. En 1968, cette conception est évidemment révolue, puisque le moment où Pedrosa écrit est celui de la recherche d'un lien nouveau entre l'artiste et la société à travers un art de la participation pourvu d'une énergie collective. C'est une motivation presque anthropologique : cet art où tous les matériaux sont possibles, où la Peinture et la Sculpture "sont niées", où la participation est fondamentale, où l'artiste sort de son isolement, fait dire à Pedrosa que « ce qui sous-tend au fond tout ce mouvement antiart est une nostalgie sacrée chez les artistes d'une société dans laquelle ils seraient aussi intégrés, aussi indispensables à la vie collective que dans les sociétés de cultures primitives, de communauté sociale authentique $»^{18}$. La teneur magique de l'art, idée que Jean-Louis Déotte nous rappelle à travers l'analyse de l'œuvre de Gilbert Simondon, comme nous le verrons plus avant, cette idée de la teneur magique de la fonction de la pensée esthétique, comme espace convergent et diffractant du technique et du religieux $^{19}$, sont ici réaffirmées et nous pouvons considérer que Pedrosa a mis le doigt sur une composante anthropologique essentielle de l'appareil artistique de cette époque.

6 L'année précédente, lors de l'importante exposition Proposta 65, qui précède d'une année Proposta 66, à la même fondation A. A. Penteado de São Paulo, le critique Pedro Geraldo Escosteguy parle de la "nouvelle esthétique » dont les œuvres exposées témoignent à ses yeux. Pour lui, l'art et l'artiste, impliqués dans une crise de l'art, se trouvent à l'époque face à un dilemne manichéen entre "l'esthétique pure ou l'engagement social $»^{20}$. Les attendus de son texte sont clairs :

Émergeant de l'historicité de l'homme dans le monde, une hypothèse rénovatrice surgit, qui suscite une nouvelle esthétique; cette dernière fait appel à une expression adéquate de l'artiste contemporain. Celui-ci répond ou ne répond pas à cet appel. Il contribue à la solution de la crise ou n'y contribue pas. Il fait œuvre de participation sociale ou une simple rêverie individuelle, selon [...] sa propre expérience de la vie, son plus ou moins grand degré de développement éthique. ${ }^{21}$

Notons que pas une ligne de ce texte de 1965 n'a perdu de sa pertinence pour un ensemble considérable d'artistes qui définissent aujourd'hui leur action en fonction de ces impératifs moraux, sociaux et politiques. L'espace de travail de l'artiste est celui $d^{\prime}$ ' une sémantique positive de protestation et de dénonciation» ${ }^{22}$. Il est clair que le contexte politique de l'année 1965 peut en partie expliquer cette affirmation, mais elle va plus loin que son contexte si l'on considère qu'aujourd'hui encore l'alternative énoncée par ce critique est pertinente dans le cas de groupes d'artistes qui entreprennent volontairement l'interaction sociale et le travail dit « engagé ». Et ceci est patent au Brésil. L'artiste qui dénonce n'est pas un artiste qui s'adapte platement ou qui renonce à lui-même et à l'art, bien au contraire. Ne pas s'adapter, c'est ne pas faire de l'art pour l'art pour " une minorité inopérante $»^{23}$. Ne pas renoncer, c'est éviter de fuir devant la complexité de la tâche qu'il y a à se maintenir dans l'art tout en protestant contre lui et son système. Se maintenir dans l'art signifie "intégrer les relations de comportement esthétique ${ }^{24}$. Comme l'ajoute Escosteguy de façon pertinente - et nous versons cela au dossier international de la problématique de la perte ou du maintien des "spécificités » dans les années 1960, dont un théoricien comme Thierry de Duve a pensé de nombreux enjeux :

Pour discerner la validité ou la permanence des valeurs intrinsèques de cette nouvelle esthétique, il est important d'analyser attentivement non seulement ce qui se présente comme entièrement neuf, mais aussi les transfigurations de traitement 
assises sur des techniques déjà expérimentées alors même qu'elles se libèrent des compromis déterminés par la tradition ou par la critique conventionnelle. ${ }^{25}$ moderniste - celle des «transfigurations dans le traitement » du médium, justement. Cette inquiétude d'un critique à l'époque où l'accélération mondiale des inventions artistiques, des nouvelles méthodes, des nouveaux matériaux, des nouvelles spatialités, de nouvelles théâtralités, de nouvelles problématiques en tous genres était croissante, est symptomatique de l'importance de la question de la relation aux techniques et aux disciplines spécifiques, et de la nécessité de les intégrer subtilement et dialectiquement dans une nouvelle dynamique esthétique.

Dans un texte de l'année 1967 sur la "Théorie de la guérilla artistique », le critique Décio Pignatari, poète concrétiste, parle de l'art d'alors comme d'une «méta-avantgarde », « consciente de soi » en tant que « processus expérimental ${ }^{26}$. Cette situation d'innovations et de modifications sensibles de l'art manifeste la conscience de soi de l'art, la conscience de ses tâches possibles, la création, écrit le concrétiste - qui n'a pas renié ses rêves de rénovation constructive de la réalité et de la société - d'une « toute nouvelle totalité $»^{27}$. Mais les conditions de l'invention sont étroitement liées à un travail à l'intérieur de " connaissances déjà codifiées ", précise Pignatari, conditions au milieu desquelles l'artiste procède à des « actes décisifs » de création ${ }^{28}$. Il s'agit ici d'une "capacité de décision et d'invention" impliquant, dans le travail de l'artiste, la «sélection et l'opération", et où «la capacité de langage " ${ }^{29}$ sous-tend l'œuvre. Sélectionner, décider, savoir utiliser les ressources pratiques et symboliques disponibles, inventer: telles sont les composantes de la guérilla artistique, de l'opération guérilla. Cela va beaucoup plus loin que ce que disait Escosteguy. Pour Pignatari, il ne s'agit plus de créer des "événements", qui sont autant de "redondances $~_{30}$ dans un art qui se contenterait d'évoluer dans son autonomie formelle - et je signale qu'Allan Kaprow définissait peu de temps auparavant le modernisme comme une machine évolutive qui se maintient à l'intérieur de l'art sans le modifier structurellement. Il s'agit de changer la structure de l'art, de faire sa vraie révolution. Pignatari prône un art de «relations $»^{31}$ et de structures révolutionnées - et non modifiées. L'art expérimental, dans ses modalités de performance, d'« event », de « happening ", etc., sera considéré comme l'un des axes de cette révolution.

L'une des singularités de l'action performative-participative est qu'elle suit une dynamique épistémologiquement multiface puisqu'on y retrouve notamment le rituel mythique et la médiation théologico-politique ${ }^{32}$. En effet, l'expérience est celle d'un récit qui s'inscrit potentiellement sur un corps individuel et, par extension utopique, collectif; le spectateur, qui est devenu "participant», est reconfiguré tant sensiblement que spirituellement («suprasensoriellement», comme disait Hélio Oiticica) au moyen d'une médiation incarnante et incorporante. Certes, nous dit JeanLouis Déotte, de tels dispositifs artistiques ne recouvrent pas l'extension du concept d'appareil. Disons que le rêve secret de tant d'artistes désireux de changer le monde et l'homme au travers de sollicitations esthétiques inclusives et incarnantes n'est pas sans visée de pouvoir, la réciproque inclusion du maitre sage (l'artiste) et du simple public qui participe constituant bien une modalité du «schéma théologico-politique de la double inclusion $»^{33}$, mais, confessons-le, par métaphore, par tension utopique vers une réalisation, de l'ordre du désir (et en fait jamais vérifiable ni normable), de quelque chose qui aurait la force de capillarité d'un appareil imperceptiblement efficient à refaire le monde. C'est exactement ce désir que Mário Pedrosa identifiait en 1968. Nous 
croyons volontiers que l'art dont nous parlons ici n'avait pas encore renoncé à une forme de pouvoir quand tant d'artistes rêvaient de transcender leurs dispositifs en une force agissante, cherchant à énoncer de nouvelles lois de l'expérience sensible, certes par la suggestion et sans enfreindre la liberté de chacun, mais aussi en consolidant la croyance en un pouvoir transformateur de l'art, en la compétence de la proposition faite par l'artiste, en sa faculté de médiation, en un mot, en son autorité. Ce que fait l'artiste brésilien Vik Muniz dans le film de Lucy Walker, Waste Land (2010) tendrait à le montrer.

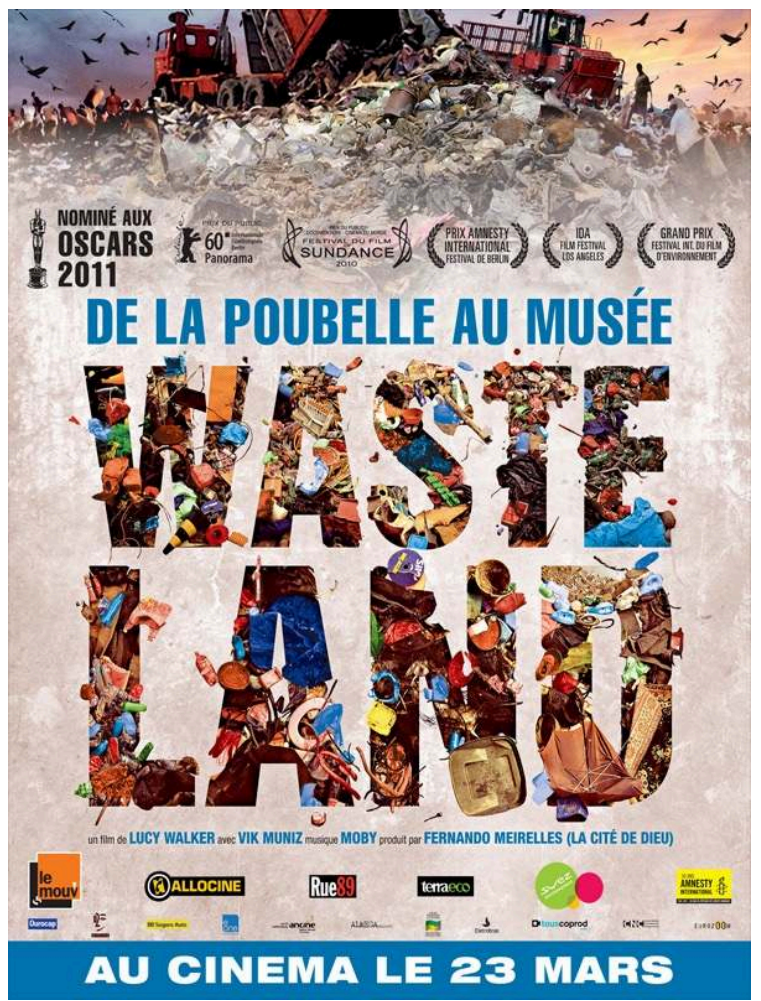

11 Vik Muniz est l'exemple même de l'artiste qui joue sur les multiples registres de l'art pour mener à bien leurs fins diverses, à moins qu'il ne s'agisse de les mener à leur fin. Il s'agit de l'histoire de l'art, de l'iconographie, de la "participation », de l'art social, du mythe de l'artiste. Sans surprise, c'est encore dans les traces du programme formulé par Oiticica en 1967 - «la volonté constructive dans le champ éthico-politico-social $»^{34}$ -, que se place apparemment Muniz. Oiticica était très clair: la "participation " implique un éventail très ouvert de pratiques, un certain chaos disciplinaire : « ce qui est constamment recherché, c'est un mode objectif de participation [...] jusqu'à fournir aux personnes présentes l'occasion de 'créer' leur œuvre $»{ }^{35}$ L'ambition de cette Nouvelle Objectivité était de promouvoir de "nouvelles conceptions d'ordre esthétique ${ }^{36}$. L'originalité de l'art brésilien fut de proposer des structures expérimentales pour le corps, dont «l'objectité » énigmatique était alors ouverte, traversée et vécue dans une dynamique relationnelle. Avec l'évolution de l'art, il est devenu possible, quelques décennies plus tard, de détourner ces pratiques de leurs sens originel. C'est ainsi que Vik Muniz produit dans Waste Land une véritable mimesis ironique des esthétiques participatives, détournant cet héritage à des fins néotraditionnelles. Le partage de l'artiste avec les participants, co-auteurs et co-artistes, dans le Jardim Gramacho de Rio de Janeiro - une immense décharge publique -, n'efface et ne dilue en rien la réalité différentielle de son statut, confirmant ainsi la 
survivance symbolique d'une représentation sociale objective. En fait, la figure de l'artiste, dont l'autorité singulière consiste dans le pouvoir de proposition et d'agencement de l'expérience artistique, n'est -en dépit des intentions et des apparences - jamais remise en cause dans les esthétiques de la «participation» collective.

Dans les années 1960, les propositions d'art de "participation » sociale cherchaient à produire un art qui élargirait et projetterait ses capacités socialement et symboliquement structurantes. Aujourd'hui, l'art brésilien est en grande partie fidèle à ce programme. Il oriente plus que jamais certaines de ses préoccupations critiques et pratiques vers la création de protocoles d'interaction et d'action communautaire avec le public. Ces "actions artistiques et activistes" sont toujours inspirées par un désir d'objectiver concrètement une philanthropie sociale. L'agenda de l'échange et de la (re)constitution de "communautés" participatives a mené de nombreux artistes « collectifs » à projeter leurs intentions relationnelles de toutes les manières possibles. Ils proposent et mettent en scène des actions éclectiques qui témoignent du désir d'exploiter toutes les tactiques d'intervention, de contact, d'interpellation, d'attention, pour " éveiller» la conscience des gens et « réanimer » leur perception, comme ils le prétendent. Ces actions sont en général urbaines. On peut considérer que la performance artistique participative constitue même une technique ready-made dans l'art brésilien, au point de parfois hypothéquer sa force originelle en des reproductions épigonales, mal structurées, à la poétique artistiquement faible, pour ne pas dire inexistante, configurant une sorte de énième mort de l'art sous les auspices de sa prétendue régénération sociale. Au Brésil, ce sont des centaines de "collectifs" d'artistes qui orientent ainsi leur travail « interactif ». Dans ce contexte d'une esthétique de la pauvreté, choisir comme objet critique l'un des artistes les plus valorisés du marché international de l'art peut sembler contradictoire. Mais le film de Lucy Walker consacré au travail de Vik Muniz est un exemple, parmi les plus paradoxaux, d'un usage de la technique de la "participation" ayant pour finalité une opération de consolidation auto-représentative du pouvoir social et iconographique de l'artiste. Le caractère altruiste de la " participation » cache la mise en scène du mythe du créateur. Waste Land obéit à un projet qui réunit quelques personnes qui ramassent et sélectionnent des ordures dans une décharge publique de Rio de Janeiro ; Muniz procède ensuite à un montage des objets sélectionnés sur le sol de grands hangars désaffectés, générant les compositions de très grande taille de la série "Pictures of Junk » (Marat assassiné de David, Atalante et Hippomène de Guido Reni, Narcisse de Caravaggio, le portrait d'un travailleur pauvre suivant le modèle d'une Madone classique et d'autres références célèbres de l'histoire de la peinture). Ces compositions gigantesques sont photographiées puis exposées comme tableaux-photographiques dans une galerie d'art. Le produit financier de la vente est reversé aux participants. Ils peuvent ainsi reconstruire leur maison, en acheter une autre, investir, changer de vie. 

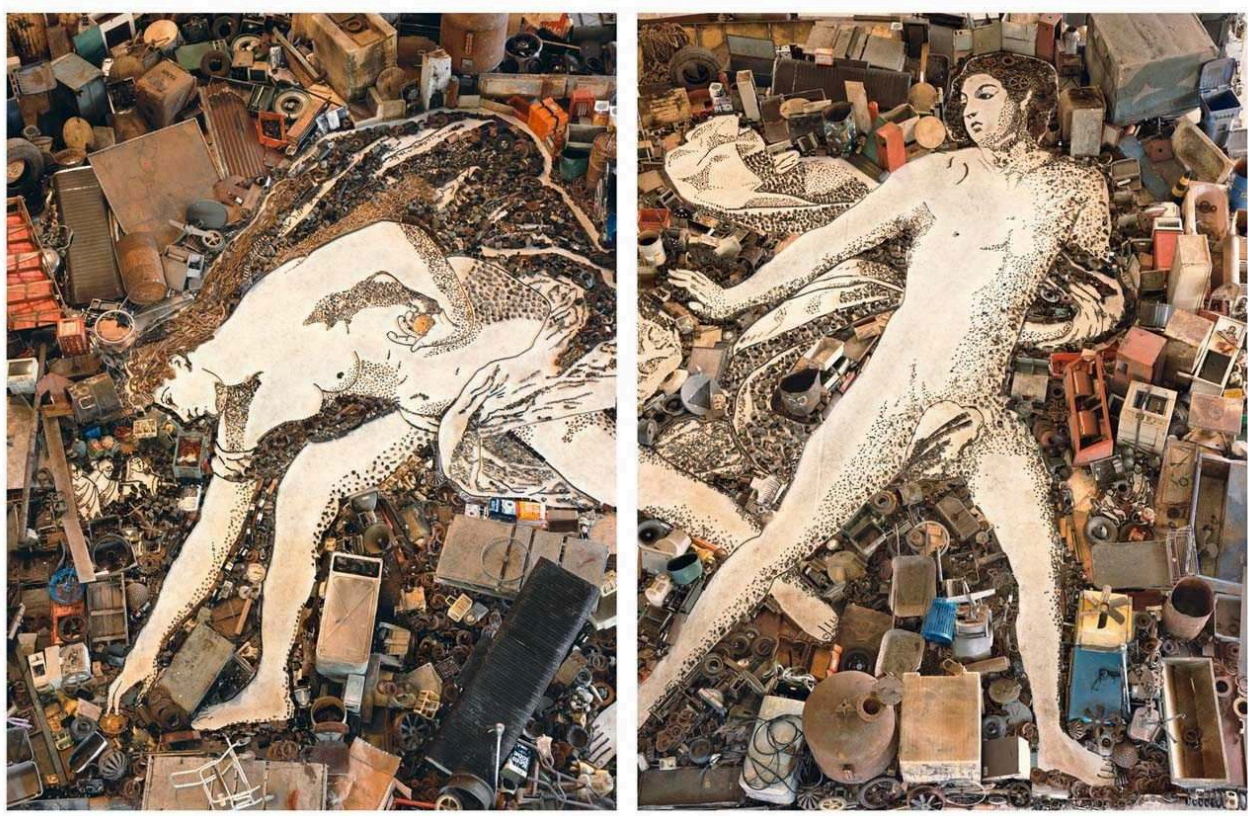

Vik Muniz, Atalante et Hippomène, d'après Guido Reni. Série « Pictures of Junk »

Depuis les années 1990, Vik Muniz produit de grands "tableaux» photographiques reconstituant certains chefs-d'œuvre de l'histoire de la peinture. La surface de ces tableaux est faite de petits fragments de papiers colorés dont l'aspect de mosaïque doit être interprété comme une évocation d'un art de la touche. L'emploi de la technique moderniste du collage trahit un usage ironique de la vision impressionniste et pointilliste. Ces collages sont ensuite photographiés et exposés en grand format. Des icônes classiques de la peinture occidentale sont aussi "reproduites" avec des techniques singulières, les Peintures au chocolat, par exemple, telle l'image de Pollock peignant au dripping, Le radeau de la méduse, etc. Le rappel de cet aspect singulier de l'iconographie munizienne est important car son œuvre est une manière permanente d'attaquer le cliché, de défaire et de refaire la valeur objective des chefs-d'œuvre de l'histoire de la peinture. Muniz ne prend pour objet et motif de ses tableaux que des valeurs sûres du patrimoine artistique. Refaisant les images à travers une mimesis paradoxale et cynique de valeurs patrimoniales qu'il maltraite et métamorphose en même temps, son esthétique de la démystification et du réenchantement conjoint du cliché est une affirmation explicite de ce que la valeur de l'art est toujours la représentation.

Une communication de Jean-Louis Déotte sur «le milieu des appareils» parle d'« un grand combat entre une figure (le géométral) et un fond mémoriel d'images historiquement datées aujourd'hui, mais qui a toujours la capacité d'alimenter d'autres combats avec d'autres figures, avec d'autres schèmes d'action $»^{37}$. Avec Muniz, ce n'est plus le géométral, à la rigueur quelque géo-métrie sensible, mais la force critique du happening et de la participation, valeurs réellement ready-made de l'art brésilien contemporain, qui entre en relation à la fois complémentaire et conflictuelle avec un fond mémoriel d'images. Pourquoi ? On peut avancer l'hypothèse que si les images de l'iconographie se voient en quelque sorte réinvesties par Muniz d'un pouvoir visuel et performatif, c'est que les pratiques relationnelles, participatives et interactives de nombreux «collectifs" tendent souvent à les négliger, à les remiser au magasin des accessoires, la responsabilité de créateur d'image paraissant soudain lourde, difficile! 
Muniz a sans doute une façon problématique de faire usage de l'image d'art, de l'introduire en des tactiques réifiantes (peut-être ne font-elles qu'objectiver la réification), mais le fait de marier opus iconographique et performance participative nous le montre en même temps dialectiquement et allégoriquement en tension avec les «activistes » qui ne veulent plus rien savoir de l'histoire de l'art et de l'histoire des images. Que l'action artistique soit participation, non plus tant à une dynamique relationnelle et intersubjective, qu'à l'image et à sa mémoire, voilà une proposition qui contraste. Le film a trois finalités : montrer, par une mise en scène très dynamique et énergique, une action sociale avec l'art; créer des images par des procédés totalement inconcevables, dans une sorte d'atelier à ciel ouvert; objectiver le pouvoir de l'artiste. La reproduction de clichés historiques à partir d'objets disqualifiés et promis à la destruction accomplit la dimension iconographique du travail, créant une aura visuelle. Les co-auteurs et co-acteurs, travailleurs pauvres, se sentent devenir des dieux et des icônes. Le film de Muniz est une sorte de happening carnavalesque à très grande échelle, distribué sur plusieurs journées de travail. Son paradoxe est de parvenir à satisfaire, sur un mode ironique et cynique, aux analyses qu'Hélio Oiticica faisait dans son essai de 1967. Ce dernier écrivait au sujet des propositions de la Nouvelle Objectivité :

Ces expériences, donc, sont importantes en tant que moyen pour introduire le spectateur non préparé dans le processus phénoménologique de révélation de l'œuvre, qui n'a plus rien à voir avec quelque chose de fermé et de lointain, mais s'offre, proposition ouverte, à sa participation totale. ${ }^{38}$

Pour Oiticica, mais aussi, à sa suite, pour tant d'artistes philanthropes évoluant dans des structures microsociales, l'artiste a encore exactement le statut symbolique du «représentant» tel que Déotte le présente comme celui qui "performe l'êtreensemble $»^{39}$. On peut en effet se demander si l'art n'est pas un appareil de «narration, révélation, délibération » certes technique, mais plus encore. Il s'agit sans doute de ce que Simondon présente comme étant «la pensée esthétique [qui] opère et juge, construisant des structures et saisissant les qualités du fond de réalité, de manière connexe et complémentaire, dans l'unité de chaque être $»^{40}$. L'art, entre religion et technique. «La pensée esthétique, restant dans l'intervalle entre la subjectivation religieuse et l'objectivation technique, se borne à concrétiser des qualités de fond au moyen de structures techniques : elle fait ainsi la réalité esthétique, nouvelle médiation entre l'homme et le monde, monde intermédiaire entre l'homme et le monde " ${ }^{41}$, écrit Simondon. L'histoire récente de l'art tend à le confirmer, surtout si elle a pour projet, comme écrit Déotte résumant Simondon, la «préservation du souvenir de l'unité et comme horizon l'unité supérieure $»^{42}$. L'art et la pensée esthétique - et nous pensons trouver dans l'action artistique brésilienne l'emblème historique d'une telle zone « est plutôt une zone de passage, dite transductive, entre phases séparées : la pensée esthétique n'est dans un premier temps ni religieuse, ni technicienne. Elle représente en effet auprès des techniques la vocation religieuse à la totalité et à la méditation et auprès de la religion la nécessité technique de l'élémentaire et de l'application. C'est ce lieu où une phase peut devenir le symbole de l'autre, comme le rappel de sa finitude, de son manque $»^{43}$.

16 Le happening filmé de Muniz répond à ce programme. La dynamique et la structure " participative » est un médium adapté à ce programme énoncé voici près de cinquante ans! Muniz maintient vivante la tradition de valorisation sociale des manifestations populaires brésiliennes - samba, fêtes collectives, Carnaval - qu'Oiticica considérait en son temps comme un modèle de mobilisation artistique et sociale. Mais ce qui fait sens 
en 2010, c'est qu'un tel théâtre populaire, caractérisé dans Waste Land par un déploiement d'énergie physique considérable de la part de tous les acteurs, avec direction et scénographie de l'artiste, met cette "participation » au service d'un jeu iconographique ironique, mais aussi au service de l'image de l'artiste, ultime convention de l'art. Waste Land est l'affirmation des pouvoirs d'agencement de la représentation comme valeur objective de l'art, l'affirmation que la production de l'image doit être considérée comme son objectité irréductible et que l'artiste en est le chef d'orchestre. Muniz exige donc que l'on pose la question de la distorsion d'une intention sociale au moyen d'un jeu qui exploite le mythe de la "participation» authentique, garante d'une expérience singulière, pour mieux réalimenter le mythe de l'artiste. Mais Waste Land exige que l'on élargisse la question selon une visée rétrospective : les intentions, déjà manifestes dans les années 1960, de faire du public un partenaire de la performance artistique, ne confirmaient-elles pas a contrario l'autorité de l'artiste? Puisqu'il crée des structures qui objectivent une proposition esthétique, ne confirmaient-elles pas déjà son pouvoir? L'agencement des forces productives par Muniz ne parle-t-il pas en toute clarté de l'impossibilité de mettre fin à son autorité? Cette impossibilité de l'artiste, pour travailler dans une certaine visibilité, à renoncer à son statut, ne contribue-t-elle pas à l'objectivation et à la permanence de son statut différentiel? En exhibant un spectaculaire pouvoir d'agencement structurel, Muniz ne souligne-t-il pas la force objectivement irréductible de la figure sociale et symbolique de l'artiste ? Ces questions sont importantes car l'on sait que le grand marché mondial des expositions est basé sur le mythe de cette figure sociale. Si le rêve de Nouvelle Objectivité participative et collective selon Oiticica est aujourd'hui pratiqué en grande partie par des artistes qui ont déjà renoncé implicitement "au règne et à la gloire », avec Muniz c'est la représentation de cette "gloire » qui est l'objet du film. Mais trahit-il en cela l'idée qu'Oiticica se faisait de l'artiste? Pas vraiment. Après toutes les déconstructions, cette figure toujours mythique est la dernière représentation à travers laquelle un mythe social se maintient, qui fait que l'art ne meurt pas. Il soutient le travail et de ceux qui prétendent diluer les pouvoirs de l'art dans le socius, en lui déniant son statut différentiel, et de ceux qui savent manipuler les prestiges de cette différence, comme Vik Muniz. La magie, fond anté-religieux et anté-technique, n'est pas loin. Nous voyons donc dans Waste Land l'allégorie baroque de ce que Déotte, analysant Simondon, décrit comme :

[...] une différenciation de la pensée magique. Laquelle ne correspond pas à un rapport du vivant à son milieu totalement indifférencié, mais déjà à une structuration de l'ensemble vivant/milieu selon le principe de la réticulation, instituant un réseau constitutif d'une pluralité d'unités. C'est le réseau qui précède les unités, c'est la réticulation qui est la clef de la bonne tension, de la bonne différence entre, à chaque fois, une figure et le fond, la figure se détachant d'un fond soit naturel, soit social-historique. ${ }^{44}$

17 Il faudrait citer en entier les paragraphes 27 et 28 de la communication de Déotte autour du nom propre et des figures-clés... Nous nous contentons d'y renvoyer, en hommage. 


\section{BIBLIOGRAPHIE}

Déotte Jean-Louis, « Au fondement de la politique et de l'art : la cosmétique », 2006, http:// www.alphabetville.org/article.php3?id_article $=49$

Déotte Jean-Louis, « Le milieu des appareils ", colloque « Le milieu des appareils », 26 et 27 octobre 2006, Maison des Sciences de l'Homme Paris Nord, Appareil, $\mathrm{n}^{\circ}$ 1, 2008, http:// appareil.revues.org/61

Ferreira Glória (dir.), Critica de Arte no Brasil: Temáticas Contemporâneas, Rio de Janeiro, Funarte, 2006

Hélio Oiticica, catalogue d'exposition, Center for contemporary art, Rotterdam / Galerie nationale du Jeu de Paume, Paris / Fundació Antoni Tàpies, Barcelona / Centro de Arte Moderna da Fundaç ão, Calouste Gulbenkian, éd. par Witte de With. Lisbon / Minneapolis, 1992-1994.

\section{NOTES}

1. Ferreira Gullar, "Arte neoconcreta: una contribuição à arte brasileira", in Critica de Arte no Brasil: Temáticas Contemporâneas, Glória Ferreira (dir.), Rio de Janeiro, Funarte, 2006, p. 60.

2. Ibid., p. 64.

3. Ibid., p. 66.

4. Loc. cit.

5. Ibid., p. 70 .

6. Loc. cit.

7. Ronaldo Brito, "As ideologias construtivas no ambiente cultural brasileiro ", Catalogue de l'exposition Projeto construtivo brasileiro na arte, Musée d'Art moderne de Rio de Janeiro / Pinacothèque de l'État de São Paulo, 1977, in Critica de Arte no Brasil: Temáticas Contemporâneas, Glória Ferreira (dir.), op. cit., p. 73-81.

8. Ibid., p. 73.

9. Ibid., p. 78.

10. Loc. cit.

11. Ibid., p. 79

12. Mário Pedroasa, "Arte ambiental, arte pós-moderna, Hélio Oiticica”, in Critica de Arte no Brasil: Temáticas Contemporâneas, Glória Ferreira (dir.), op. cit., p. 144.

13. Hélio Oiticica, "Situação da Vanguarda no Brasil", catalogue de l'exposition Proposta 66, FAAP, São Paulo, 1966, in Critica de Arte no Brasil: Temáticas Contemporâneas, Glória Ferreira (dir.), op. cit., p. 147.

14. Ibid., p. 148.

15. Ibid.

16. Mário Pedrosa, "Do porco empalhado aos critérios da critica", in Critica de Arte no Brasil: Temáticas Contemporâneas, Glória Ferreira (dir.), op. cit., p. 209.

17. Loc. cit.

18. Loc. cit.

19. Jean-Louis Déotte, "Le milieu des appareils », colloque «Le milieu des appareils », 26 et 27 octobre 2006, Maison des Sciences de l'Homme Paris Nord, Appareil, n 1, 2008, http:// appareil.revues.org/61 
20. Pedro Geraldo Escosteguy, "No limiar de uma nova estética”, catalogue de l'exposition Proposta 65, FAAP, São Paulo, 1965, in Critica de Arte no Brasil: Temáticas Contemporâneas, Glória Ferreira (dir.), op. cit., p. 137.

21. Loc. cit.

22. Loc. cit.

23. Loc. cit.

24. Loc. cit.

25. Ibid., p. 138.

26. Décio Pignatari, “Teoria da guerrilha artística", Critica de Arte no Brasil: Temáticas Contemporâneas, p. 158-159.

27. Ibid., p. 160.

28. Loc. cit.

29. Loc. cit.

30. Ibid., p. 159.

31. Loc. cit.

32. Ces catégories sont synthétisées lapidairement dans une communication dense de Jean-Louis Déotte intitulée «Au fondement de la politique et de l'art: la cosmétique », 2006, http:// www.alphabetville.org/article.php3?id_article $=49$

33. Loc. cit.

34. Hélio Oiticica, «Schéma général de la nouvelle objectivité », in Hélio Oiticica, catalogue d'exposition, Center for contemporary art, Rotterdam / Galerie nationale du Jeu de Paume, Paris / Fundació Antoni Tàpies, Barcelona / Centro de Arte Moderna da Fundação, Calouste Gulbenkian, éd. par Witte de With. Lisbon / Minneapolis, 1992-1994, (note 4), p. 117.

35. Ibid., p. 116.

36. Ibid., p. 117.

37. Jean-Louis Déotte, « Le milieu des appareils », op. cit., paragraphe 10.

38. Hélio Oiticica, "Schéma général de la nouvelle objectivité », in Hélio Oiticica, catalogue d'exposition, op. cit., p. 118.

39. Jean-Louis Déotte, " $\mathrm{Au}$ fondement de la politique et de l'art : la cosmétique », op. cit.

40. Gilbert Simondon, Le mode d'existence des objets techniques, (1958), Paris, Aubier, 1969, p. 190. Je signale que les citations 40 et 41 proviennent de l'article de Jean-Louis Déotte, «Le milieu des appareils ", op. cit.

41. Ibid., p. 182.

42. Jean-Louis Déotte, « Le milieu des appareils », op. cit., paragraphe 20.

43. Loc. cit.

44. Ibid., paragraphe 13 .

\section{RÉSUMÉS}

Depuis les années 1960, l'art brésilien est caractérisé par une présence importante de pratiques qui cherchent à instaurer une relation esthétique singularisante avec le public. Du néoconcrétisme jusqu'à aujourd'hui, la «participation » est devenue un véritable genre qui n'a pas perdu de sa force mythique de motivation. L'action artistique participative continue d'être pensée comme une tactique de rénovation de l'expérience sensible. Quelques textes fondateurs 
des années 1960, notamment ceux de Hélio Oiticica, en définissent la teneur. Ils suscitent encore aujourd'hui de puissants échos. Mais la limite entre la force originelle et l'usure est ténue. Un film comme celui que Lucy Walker a consacré en 2010 au travail de Vik Muniz, Waste Land, démontre comment il est possible de faire usage de la participation, menée sur une échelle inédite, pour la mettre au service de fins multiples et ambiguës qui confirment notamment le pouvoir magique et hyper-représentatif de l'artiste, alors même qu'il feint de se dissoudre dans le partage de l'action. La présence culturelle et la durée de cet idéal représentent dans l'histoire de l'art une force structurante, un appareil productif qui confirme la singularité de la pensée esthétique quand celle-ci articule visées éthiques et techniques du corps.

Since the 1960s, Brazilian art is characterized by a significant presence of practices which try to establish a singularizing aesthetic relationship with the public. From Neoconcretism until today, "participation" has become a genre that has not lost its mythical motivating force. The participative artistic action continues to be thought of as a tactics of renovation of the sensitive experience. Some founding texts of the 1960s, in particular those of Hélio Oiticica, define its content. They arouse even today powerful echo. But the boundary between the original strength and the wear is tenuous. A movie as the one that Lucy Walker dedicated 2010 to the work of Vik Muniz, Waste Land, demonstrates how it is possible to use of the patent of participation, led on an unprecedented scale, to attend some ambiguous purposes which confirm in particular the magic and hyper-representative power of the artist just when it pretends to dissolve in the sharing of the action. The cultural presence and the duration of this ideal represent a structuring strength, a production apparatus which confirms the peculiarity of the aesthetic thought when this one articulates ethical aims and bodily technics.

\section{INDEX}

Mots-clés : art brésilien, (néo)concrétisme, participation, artiste (mythe de l'), image, représentation

\section{AUTEUR}

\section{STÉPHANE HUCHET}

Professeur à l'École d'Architecture / École des Beaux-Arts, Université fédérale de Minas Gerais, Belo Horizonte, Brésil, histoire et théorie de l'art et de l'architecture, st.huchet@gmail.com 